\title{
Intra-operative Imaging for Brain Tumour Resection in Paediatric Patients
}

\author{
Christopher Parks, ${ }^{1}$ Shivaram Avula, ${ }^{2}$ Laurence J Abernethy, ${ }^{2}$ Elizabeth J Wright ${ }^{3}$ and Conor L Mallucci
}

1. Consultant Paediatric Neurosurgeon; 2. Consultant Paediatric Neuroradiologist; 3. Consultant Paediatric Anaesthetist,

Alder Hey Children's NHS Foundation Trust, Liverpool, UK

\begin{abstract}
There is currently significant investment by healthcare providers into intra-operative magnetic resonance imaging (ioMRI). It is an expensive technology, but due to its proven benefit in tumour resection it is becoming the gold standard of care in brain tumour resection. Alder Hey Hospital has routinely used this technology since 2009. In our department, ioMRI has been used in approximately 130 cases. These consist predominantly of resective tumour cases but also include biopsies, epilepsy surgery and other complex cases. In tumour resections $32 \%$ had further resection following iOMRI under the same anaesthetic. Unnecessary early return to theatre has been reduced from $14 \%$ prior to the use of iOMRI to $0 \%$ within 6 months of surgery. The adoption of this technology as an adjunct to paediatric neurosurgery has led to a significant improvement in the service delivered. It is possible that the application of advanced MRI techniques will improve this even further.
\end{abstract}

\section{Keywords}

Intra-operative imaging, intra-operative magnetic resonance, iOMRI, paediatric brain tumour, 3Tesla, advanced MRI

Disclosure: The authors have no conflicts of interest to declare.

Received: 19 September 2013 Accepted: 15 November 2013 Citation: European Neurological Review, 2013;8(2):159-63 DOI:10.17925/ENR.2013.08.02.159 Correspondence: Christopher Parks, Consultant Paediatric Neurosurgeon, Alder Hey Children's NHS Foundation Trust, Eaton Road, West Derby, Liverpool, L12 2AP, UK. E: cjcparks@gmail.com

A number of paediatric and adult neurosurgical units are investing significant resources in the development of intra-operative magnetic resonance imaging (iOMRI). In Alder Hey Hospital we have been fortunate enough to have this service available since october 2009 and so we have accumulated significant experience in this field. In our institution it is used routinely as an adjunct in the resection of paediatric brain tumours, in lesional epilepsy surgery and in other challenging cases.

Investment in this expensive technology has been driven by the improvement in prognosis gained by more complete primary resection. In paediatrics, low-grade glioma is the most common tumour and total resection can be curative without the need for adjuvant therapy but improved degree of resection has also been shown to be a major prognostic factor in high-grade tumours, such as medulloblastoma, high-grade glioma and ependymoma. ${ }^{1-3}$

\section{Components of Intra-operative Magnetic Resonance Imaging}

MRI has been widely available for many years in neurosurgical units, but there are significant considerations when utilising this technology for intra-operative imaging. The choice of technology is the main consideration at the planning stage in terms of structural set-up of the theatre that will be used and budgeting for a costly component of the service. At Alder Hey, we have a three Tesla (3T) (high-field) MRI scanner utilising a 'two-room solution' (see Figure 1). This enables the use of the scanner for diagnostic imaging while the theatre is in use, but not requiring ioMRI. This ability to use the two components independently makes the facility economically viable. Not all theatre complexes would be able to house a 3T scanner due to the weight, the installation and the screening required. In such institutions options would include opting for a low-field imaging solution or accepting the inherent problems of using a theatre separated from the normal theatre complex. Current high-resolution ultrasound equipment is safe and gives accurate real-time information about resection margins but it will not be able to replace the need for MRI. 4,5 It may, however, be a reasonable alternative to low-field systems that have a low signal-to-noise ratio (SNR)

If a two-room solution is adopted the major components are the scanner, an operating table with head clamp and a transfer system. Considerations with each of these components are outlined below.

\section{Magnetic Resonance Scanner}

Our department is equipped with a Philips Achieva ${ }^{\circledR}$ 3-T scanner (Philips Healthcare, Best, Netherlands) with a length of $157 \mathrm{~cm}$ and an inner bore of $65 \mathrm{~cm}$. The size of the inner bore is important as the patient needs to fit in with a head coil on. This does normally not pose any problems in the neutral supine position but becomes more challenging in prone surgery with the patient's neck flexed and retracted for posterior fossa surgery. The use of a $3 \mathrm{~T}$ system allows advanced imaging and has a high SNR, which is especially important in small, immature brains of infants. However, the higher field strength does increase the magnetic susceptibility and chemical shift artefacts are increased. This will be discussed further in the next section.

\section{Head Clamp and Coils}

Cranial surgery using optical navigation requires a head clamp in order to maintain the position of the patient's head in relation to a reference array. With an ioMRI all components leaving the theatre to enter the MRI suite must be MRI compatible. Coils around the patients 


\section{Figure 1: Two-room Solution Intra-operative Magnetic Resonance Imaging Suite}
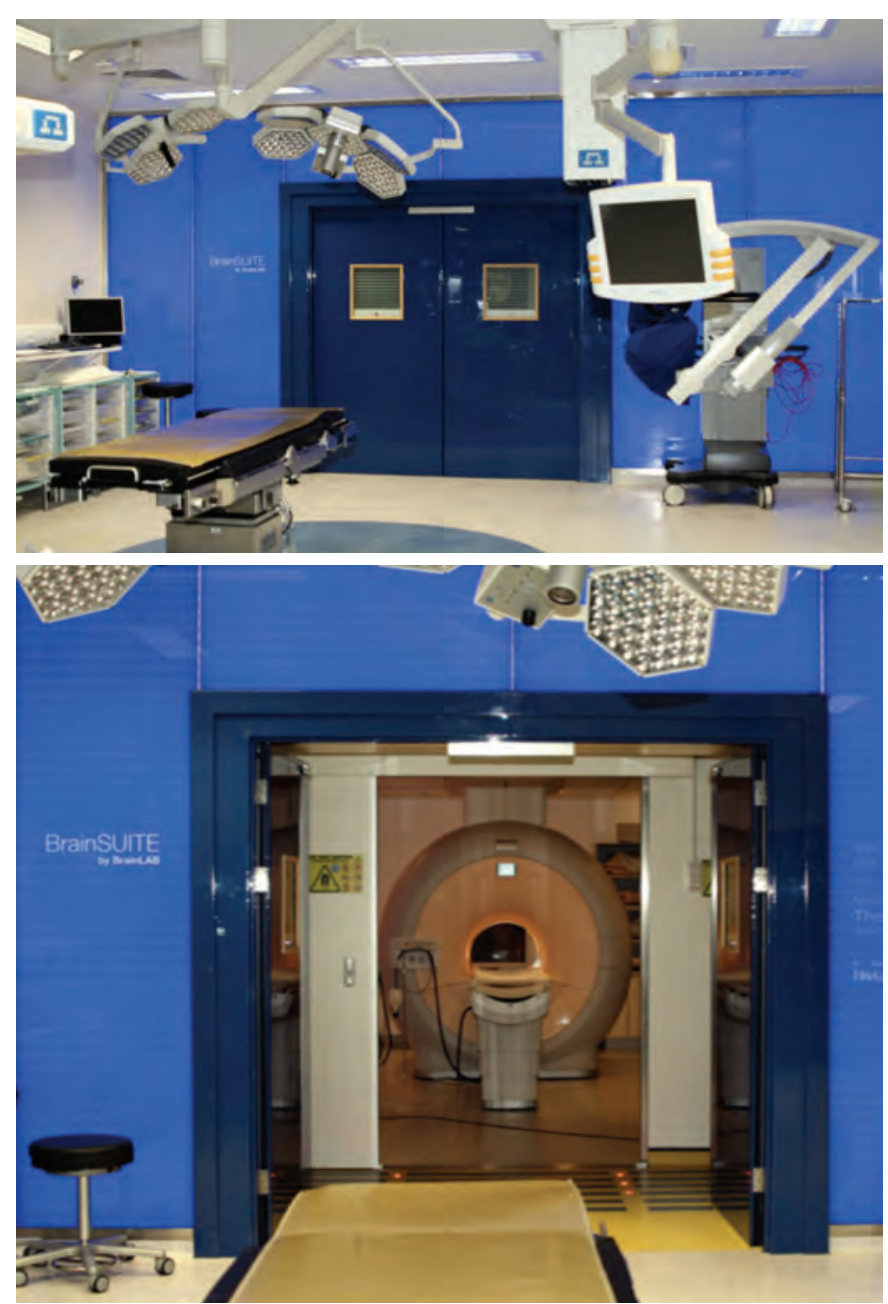

Operating theatre (top) and view through to MRI scanner (bottom)

head are required to enable scanning, but must maintain the sterility of the operative field. It is possible to use a carbon fibre head clamp and parallel flexible coil placed over the drapes, but better results are obtained using a dedicated intra-operative head coil (NORAS MRI Products, Hochberg, Germany).

The NORAS 8-channel inter-operative head coil has an integral fivepin head clamp and 14 fiducial markers, which enables automatic registration of the navigation system to the new ioMR images following a scan (see Figure 2).

\section{Transfer System}

The patient is positioned for their procedure on a theatre table that enables the normal changes in position required by a surgeon during surgery, but is compatible with a transfer trolley that can move the patient in the head clamp to the MR scanner. Alder Hey uses a Maquet system (Maquet, Rastatt, Germany), which docks securely with the operating table and the MR table allowing the patient to slide from one table to the next while remaining in the head clamp.

\section{Considerations in Use Preparation}

Prior to performing surgery, when ioMRI is intended, the radiographers, theatre staff, anaesthetist and radiologist should be informed. This is to make certain that the appropriate planning is put in place to ensure sufficient experience and availability of staff in order to avoid delays with the patient under anaesthesia.

The anaesthetist has several considerations to bear in mind due to the fact that access to the patients head is difficult after starting surgery. For this reason, the endotracheal tube should be fixed securely prior to positioning. All lines and monitoring equipment should either be MRI compatible or be able to be removed prior to ioMRI. We would advise the use of MRI compatible drip stand and drug infusion pumps from the start of the case so that there is no need to interrupt drug delivery by changing systems for the scan. Conventional electrocardiograms (ECG) electrodes, diathermy plate and temperature probes must be removed and so should be placed with this in mind at the start of the anaesthetic. Vigilance to avoid leaving equipment, such as laryngoscope blades, on the operating table is equally important.

A patient must be positioned for surgery with consideration of the use of the ioMRI facility. For example, the use of a sitting position of the patient would not be compatible as in our experience we have not found this to limit surgical access. The patient's head should be placed so that the area to be scanned will be at the isocentre of the magnetic field. On occasion with larger patients flexed in the prone position a trial transfer is necessary to ensure that they would fit into the magnet with optimal positioning. Patients should be draped using disposable surgical drapes without the use of metallic clips to secure them. We suture the drapes in place around the incision site in order to avoid inadvertent movement of the drapes during transfers, which could reduce sterility of the operative field.

\section{Transfer}

once a decision has been made to perform an intra-operative scan the radiographers should be pre-warned so that they can finish outpatient diagnostic imaging and prepare and clean the scanner. This is performed using a broad-spectrum disinfectant active against viruses, fungi and bacteria.

Haemostasis should be achieved by the surgeon as even a small ooze of blood can cause significant blood loss during the scan as well as interfering with interpretation of the imaging. We would advise avoiding the use of haemostatic agents as they can make image interpretation more difficult. If they are required, a note should be made as to where they are placed so that this can be factored in when reviewing images.

The cavity should be filled with Ringers lactate solution or saline to avoid the artefact from air in the cavity. Retractors are all removed and the wound is usually tacked closed and then covered with an occlusive adhesive cover (Ioban ${ }^{\mathrm{TM}}, 3 \mathrm{M}$, Minnesota, US). The whole operative field is covered with a further disposable drape and the image guidance reference array is removed if it is not MRI compatible. After this, the surgeon can remove their sterile gown and gloves. The disposable drapes are then cut leaving a smaller draped area around the surgical site. The operating table is placed in a neutral position ready for transfer of the patient and all ferromagnetic objects are removed or replaced with MRI-compatible ones. The surgical instruments are all checked and counted before being covered to preserve sterility.

The anaesthetist will need to enter the MRI suite with the patient and so should remove all ferromagnetic objects from their person. We would advise the use of surgical scrubs without pockets to facilitate this. Incorporation of wave guides in the walls of the MRI suite allows 
connection to external monitoring equipment that is not MRI compatible. The doors to the adjacent MRI suite can then be opened and transfer and imaging performed

\section{Possible Sources of Artefact}

As our experience with ioMRI increases, so does our experience of causing, identifying and, therefore, preventing imaging artefact (see Figure 3)

There will be expected artefacts from head-holding pins even though they are titanium and considered MRI compatible. This is not normally a concern as the pins are normally distant to the area to be resected. It is now possible to use carbon pins if it were felt pre-operatively that this would cause problems (see Figure 4).

The spring inside the cuff valve of an endotracheal tube should be outside the MRI head coil as it can cause significant distortion of images.

Air in the head cannot always be avoided if the incision is not at the uppermost part of the head. This can cause brain shift, which can make interpretation of imaging difficult. It is important to compare closely with pre-operative imaging to note the position of a lesion in relation to sulci, gyri and other identifiable structures. Air will also significantly affect gradient and echo-planar sequences.

Intracranial haemorrhage, and even blood pooled on the coil beneath the head, has been known to cause a curved artefact on images.

\section{Experience and Results \\ Tumours}

The series of 79 tumour resections performed in 73 children between October 2009 and January 2012 has been previously published. ${ }^{6}$ In this series the mean age was 9.5 years. Overall, ioMRI led to further surgery in 25 cases (32\%)

Complete resection was the surgical intent in $47 / 79$ (59\%) of cases. This was achieved in $57 \%$ after ioMRI and the other patients either had further resection of residual tumour or a second look at equivocal areas on the scan. In all, 34/47 (72 \%) achieved complete resection.

Partial resection was intended in 32/79 (41\%) of cases. These lesions were predominantly midline chiasmatic/hypothalamic, brainstem gliomas or high-risk craniopharyngiomas involving the hypothalamus and surgical intent was subtotal resection in order to preserve vital or eloquent structures. This strategy was decided prior to surgery in a multi-disciplinary term review. Surgical resection was extended in 13/32 (41 \%) of operations following ioMRI. In these patients, the resection could be maximised while preserving important structures.

Our most recent publication ${ }^{7}$ is a comparison of 36 cases where ioMRI was used in surgery to a similar cohort of patients operated on prior to the availability of ioMRI. The diverse pathology encountered is outlined in Table 1. All cases included had a pre-operative intent of complete resection. In the group where ioMRI was not available, $14 \%$ underwent repeat resection within 6 months of surgery. In the ioMRI group, 11/36 (30\%) patients had unequivocal residual disease on ioMRI and 10/11 underwent further resection under the same anaesthetic. None of this patient group required repeated resection in the following 6 months. Since the use of ioMRI in Alder Hey there has been no avoidable early second-look procedures for residual disease.

\section{Figure 2: Integrated Head Clamp and Magnetic Resonance Imaging Coil}
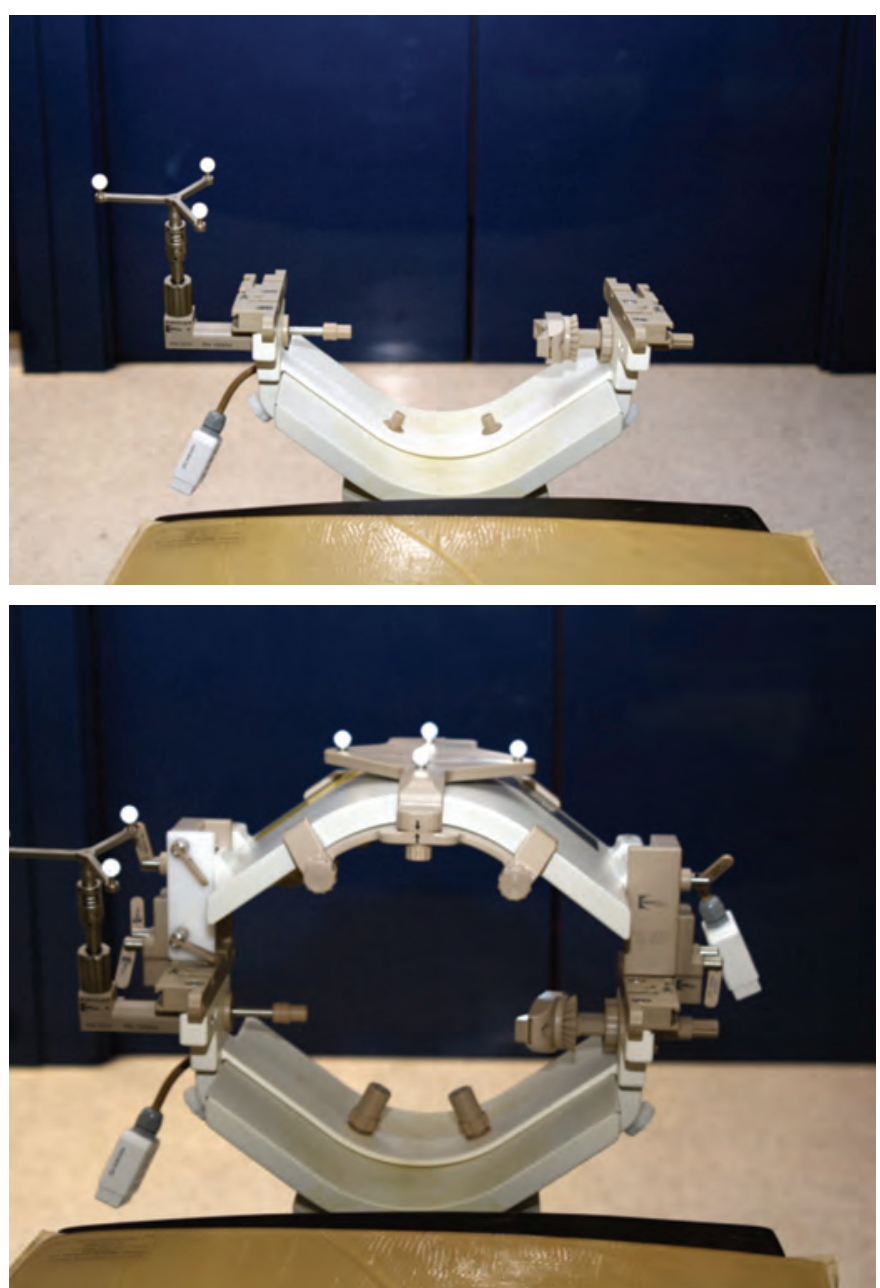

Figure 3: Identifying Artefacts

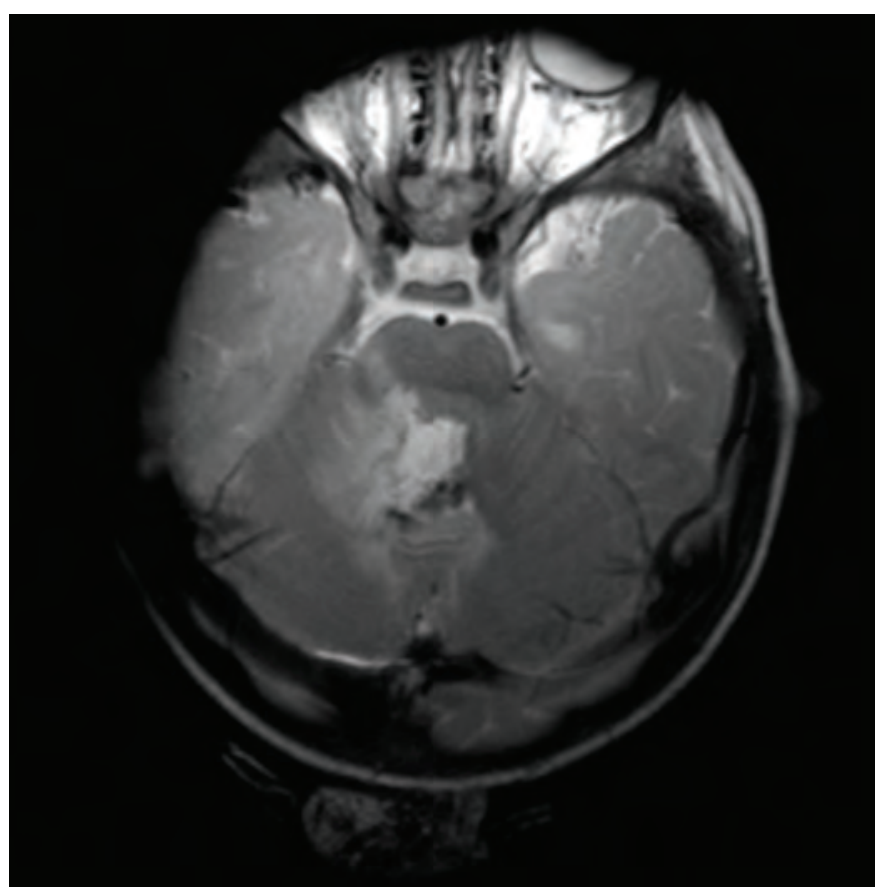

It can be difficult to identify the cause for significant artefacts. A systematic approach should be adopted as the cause can be as simple as not connecting part of the head coil as shown in this image. 


\section{Figure 4: Carbon Head Pins}

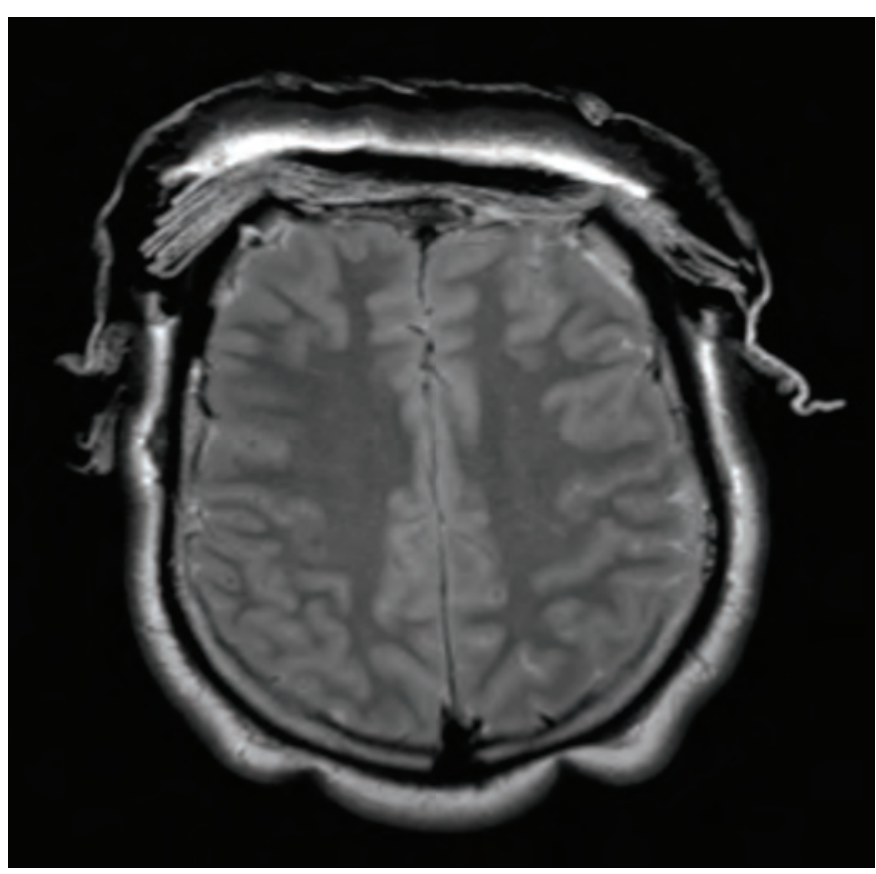

The indent of the pins can be seen posteriorly without the usual artefact expected from titanium pins. This is usually not a concern but could be important if an area of interest was close to the pin site.

\section{Table 1: Comparison of Tumour Pathology}

\begin{tabular}{lll}
\hline Pathology & Pre ioMRI Group & ioMRI Group \\
Grade I/II & 28 & 27 \\
Pilocytic astrocytoma & 12 & 12 \\
\hline Other low-grade gliomas & 3 & 9 \\
\hline Ependymoma (WHO II) & 5 & 4 \\
\hline Pituitary adenoma & 2 & 1 \\
\hline DNET & 1 & 0 \\
\hline Craniopharyngioma & 2 & 0 \\
\hline Choroid plexus papilloma & 1 & 0 \\
\hline Ganglioglioma & 1 & 0 \\
\hline Schwannoma & 1 & 1 \\
Grade III/IV & 7 & 8 \\
PNET & 4 & 3 \\
\hline ATRT & 1 & 1 \\
\hline Anaplastic ependymoma & 1 & 3 \\
\hline Anaplastic astrocytoma & 2 & 0 \\
Others & 1 & 1 \\
Pineal papillary tumour & 1 & 0 \\
\hline Vascular malformation & 0 & 1 \\
\hline This table highights the bag & &
\end{tabular}

This table highlights the broad spectrum of pathology encountered by paediatric neurosurgeons. The lesions are predominantly low grade. This table is reproduced from our department's previously published study. ${ }^{7}$ ATRT = atypical teratoid rhabdoid tumour; DNET = dysembryoplastic neuroepithelial tumour; ioMRI = intra-operative magnetic resonance imaging: $P N E T=$ primitive neuroectodermal tumour; $W H O=$ World Health Organization.

\section{Other Experience}

In Alder Hey we have used the ioMRI in approximately 130 cases. These additional cases are predominantly more resective tumour cases but the facility is being more widely utilised as our experience grows.

In lesional epilepsy surgery the benefits are similar to those for standard tumour surgery, but it has also proved useful in disconnective surgery where due to the complex 3D anatomy it is helpful to ensure that the surgical intent has been achieved.8, This again avoids the potential need for second-look procedures. In procedures where the resection material does not look any different from normal brain (cortical dysplasia, for example) and the accuracy of image guidance is of paramount importance, ioMRI can be used to update the neuronavigation and so reduce errors due to brain shift.

Young patients having temporal lobe surgery avoid the need for a second anaesthetic for post-operative MRI by performing the imaging under the same anaesthetic in the ioMRI facility. This has become the standard process for all temporal lobe cases now enabling the surgeon to be sure that surgical intent has been achieved prior to the end of surgery.

If a brain lesion is to be biopsied the facility can be useful too. When a patient is prone it can be difficult to accurately register neuronavigation as the face is the main distinguishing feature and can be difficult to access. In such cases we would perform an MRI and automatic registration following positioning of the patient prior to skin preparation and draping. Following biopsy it has now become routine to perform post-operative imaging under the same anaesthetic to confirm the biopsy tract has taken tissue from the area of abnormality. This is invaluable if a histology result is non-diagnostic as any remaining doubt as to whether a small lesion was accurately sampled is removed completely. A short sequence of images, such as a fluid attenuated inversion recovery (FLAIR), is normally satisfactory to display this and adds little time to the procedure.

When performing complex anterior approaches to decompress the upper spinal cord and brainstem by removing clival lesions or treating $\mathrm{C} 2$ abnormalities the ability to use neuro-navigation is helpful with a scan performed with the patient in the operative position. The iOMRI can also demonstrate adequate decompression and achievement of surgical goals.

\section{Complications}

In our patients we have not experienced any change in our infection rate due to the use of ioMRI despite the lengthening of procedures and moving from the theatre to MRI with open (but covered) wounds.

Post-operative haematoma is also less of a concern following tumour resection as all patients have a scan following surgery, which would show evolving haematoma if it were to occur. Obviously late bleeding is still possible and should be considered with signs of deterioration but has not been a problem in our patient group.

\section{Discussion}

High-field ioMRI in its current format is rapidly becoming the gold standard of care for the operative management of paediatric brain tumours. It prevents unnecessary return to theatre for removal of residual tumour and obviates the need for post-operative MRI under anaesthetic in the days following resection. It may well reduce post-operative neurological deficit following surgery in eloquent areas and when coupled with neuro-navigation improves resection rates and, therefore, prognosis. As understanding of tumours improves and scanning technology develops it is possible that the benefits will further increase.

Alternative technology exists to aid maximal resection of brain tumours. The use of 5 aminolevulinic acid (5-ALA) fluorescence has been proved to be helpful in high-grade glioma resection, but remains off-licence and of limited benefit in the most commonly occurring paediatric brain tumours. ${ }^{10}$ Ultrasound resolution is now good but cannot possibly match that of a $3 \mathrm{~T}$ MRI scan. The former has significant benefits in 
terms of speed, real-time imaging and cost but it is sometimes difficult to distinguish tumour from oedema surrounding the resection cavity. Ultrasound will never replace post-operative MRI imaging to establish the extent of resection and a baseline prior to ongoing treatment.

\section{Intra-operative Advanced Magnetic Resonance Imaging}

The use of advanced MRI is discussed by Abernethy et al. ${ }^{11}$ High-field MRI has the benefit that it can provide functional and biochemical as well as anatomical information to the surgeon. Techniques such as diffusion tensor imaging (DTI) and fibre tracking have been applied to show the position of vital tracts in close proximity to a resection margin, which enables a surgeon to maximise resection while avoiding post-operative deficit.

On occasions it has been beneficial to apply a spectroscopy voxel to an equivocal area of resection margin and compare this to pre-operative spectroscopy results. This has led the surgical team to decide not to perform further resection at the margin where distinguishing operative changes from residual disease can be challenging (see Figure 5).

It is likely that the use of perfusion imaging utilising arterial spin labelling (ASL), which does not require gadolinium infusion, will become increasingly useful as processing of the data improves.

\section{Immeasurable Service Improvement}

While there are significant benefits of ioMRI that can be measured, evaluated and published, the benefits extend beyond this. As a surgeon, one of the most palpable merits is when, at the end of a long

\section{Figure 5: The Use of Intra-operative Magnetic Resonance Spectroscopy}

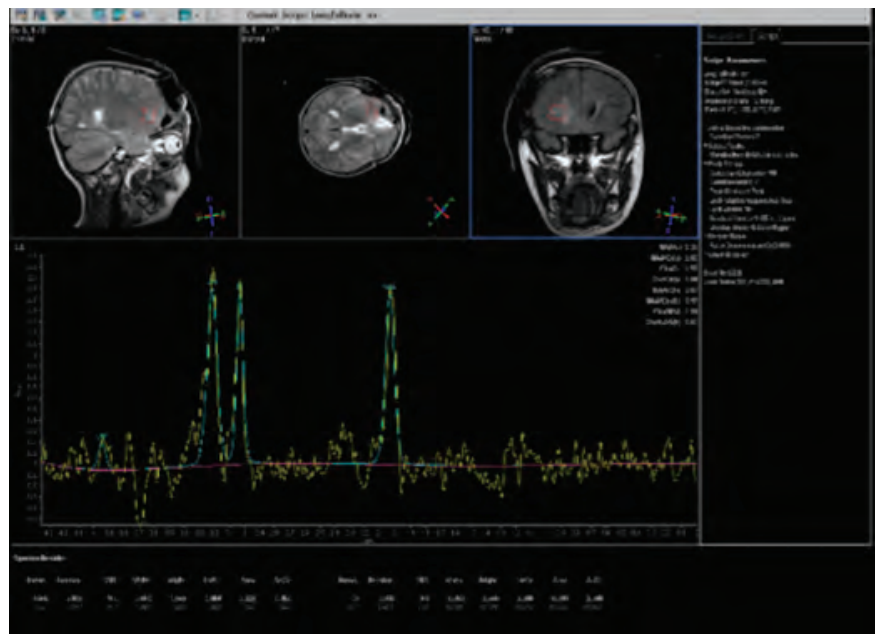

The T2-weighted images showed areas of hyperintensity posterior to the surgical cavity, some of its extending to the splenium of the corpus callosum and some close to the right caudate nucleus and internal capsule. Single voxel spectroscopy focused over this region of signal abnormality showed reduced $\mathrm{N}$-acetylaspartate peak but no significant elevation of the choline peak, more in keeping with oedema than neoplastic change.

and complex procedure, one can find a patient's parents and say that their child's surgery has gone well and that the brain tumour has been completely removed and not then have to follow with a caveat that we will check with a scan within the next 48 hours. The relief of parents and the positive effect on the patient journey is impossible to measure and cost out but is easy to feel.
1. Albright AL, Wisoff JH, Zeltzer PM, et al., Effects of medulloblastoma resections on outcome in children: a report from the Children's Cancer Group, Neurosurgery, 1996;38:265-71.

2. Finlay $\mathrm{JL}$, Wisoff $J \mathrm{H}$, The impact of extent of resection in the management of malignant gliomas of childhood, Childs Nerv Syst, 1999;15:786-8.

3. Rodríguez D, Cheung MC, Housri N, et al., Outcomes of malignant CNS ependymomas: an examination of 2408 cases through the Surveillance, Epidemiology, and End Results (SEER) database (1973-2005), J Surg Res, 2009; 156:340-51.

4. Ivanov M, Wilkins S, Poeata I, Brodbelt A, Intraoperative ultrasound in neurosurgery - a practical guide, Br J Neurosurg,
2010;24:510-17.

5. Mair R, Heald J, Poeata I, Ivanov M, A practical grading system of ultrasonographic visibility for intracerebral lesions, Acta Neurochir, 2013:155:2293-8.

6. Yousaf $\lrcorner$, Avula $S$, Abernethy $\sqcup$, Mallucci $C L$, Importance of intraoperative magnetic resonance imaging for pediatric brain tumor surgery, Surg Neurol Int, 2012;3(Suppl. 2):S65-72.

7. Avula S, Pettorini B, Abernethy L, et al., High field strength magnetic resonance imaging in paediatric brain tumour surgery-its role in prevention of early repeat resections, Childs Nerv Syst, 2013;29:1843-50.

8. Sommer B, Grummich P, Coras R, et al., Integration of functional neuronavigation and intraoperative MRI in surgery for drug-resistant extratemporal epilepsy close to eloquent brain areas, Neurosurg Focus, 2013:34:E4.

9. Buchfelder M, Ganslandt O, Fahlbusch R, Nimsky C Intraoperative magnetic resonance imaging in epilepsy surgery, Magn Reson Imaging, 2000:12:547-55.

10. PreuB M, Renner C, Krupp W, et al., The use of 5-aminolevulinic acid fluorescence guidance in resection of pediatric brain tumors, Childs Nerv Syst, 2013;29:1263-7.

11. Abernethy $\amalg$, Avula S, Hughes GM, et al., Intra-operative 3-T MRI for paediatric brain tumours: challenges and perspectives, Pediatr Radiol, 2012;42:147-57. 